\title{
Positioning system for assembly and manufacturing tasks
}

\author{
Radu-Eugen Breaz ${ }^{1, *}$, and Sever-Gabriel Racz ${ }^{1}$ \\ ${ }^{1}$ Lucian Blaga University of Sibiu, Department of Industrial Machines and Equipment, Engineering \\ Faculty, Emil Cioran 4, 550025 Sibiu, Romania
}

\begin{abstract}
The paper presents a two-axis positioning system using asynchronous motors as actuation devices. The use of asynchronous motors reduces the overall cost of the system, while providing actuating torques superior to the ones provided by stepping motors. The system is controlled by mean of a programmable logic controller (PLC) and two voltage-frequency inverters fitted with motion control cards. In contrasts to a stepping motor system, which works in open loop mode, the proposed system uses closed loop control on each axis. The motion loop is closed by means of an incremental encoder.
\end{abstract}

\section{Introduction}

Industrial activities are mainly based upon performing a controlled set of technological and non-technological movements. Consequently, motion control tasks must be unfolded on every manufacturing and/or assembly system. To achieve that goal, various types of motion control systems and consequently various types of actuation devices are used.

A special type of applications is considering low-cost solutions, yet providing enough accuracy and degree of control, for such kind of systems. Stepping motors, working in open loop control systems are widely used as actuation devices for low-cost motion control applications due to their ease of control and, of course, due to their reduced cost and wide availability. Even this approach is well-known, new approaches and control strategies [1-2] have lately improved the performances of stepping motors-based applications, making them comparable, in terms of accuracy with closed loop systems working with much more expensive actuation devices, such as dc servomotors or even synchronous servomotors.

However, for industrial manufacturing and assembly task, usually the torques provided by stepping motors are too small. Alternatively, asynchronous motors, also referred as induction motors could be used as actuation devices for low-cost motion control systems, because they provide torques superior to the ones provided by stepping motors [3]. Recent advancements have further improved the performances of low-cost motion control systems with induction motors [4-7].

Previous work of using induction motors as actuation devices in custom-built motion control systems for performing various technological operations (incremental forming being one of these) were reported [8-9] by members of the authors research team.

\footnotetext{
* Corresponding author: radu.breaz@ulbsibiu.ro.
} 


\section{Induction motor as actuation device in motion control systems}

A typical motion control structure using induction motor as actuation device is presented in figure 1. It involves a single closed loop for controlling the speed of the motor and the velocity of the final element of the transmission system. The speed feedback is provided by an incremental encoder (the speed information is obtained by measuring the frequency of the pulses emitted by the encoder), to keep the price of the system as low as possible. The process of providing the motor with the currents and voltages required by the speed regulation algorithms is handled by a vector-controlled voltage/frequency inverter [3].

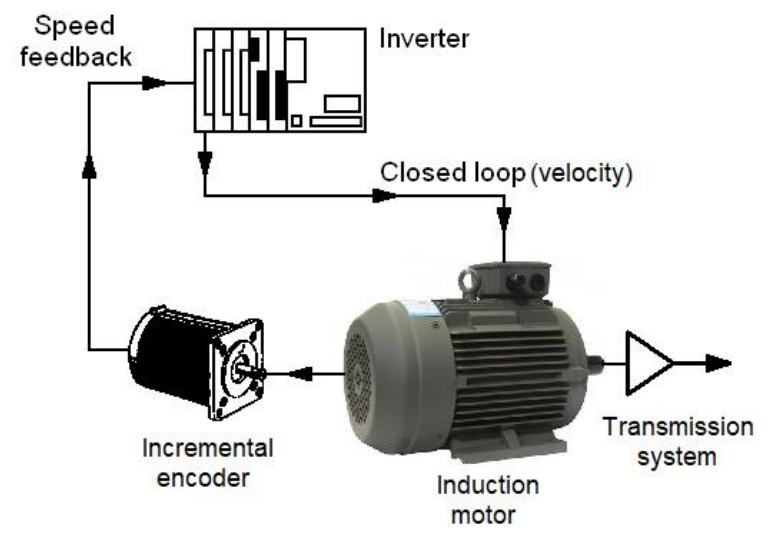

Fig. 1. Closed velocity loop with induction motor as actuation device.

By adding a motion control card as a supplementary module to the voltage/frequency inverter and using both the number of pulses emitted by the encoder and their frequency as feedback signals, as presented in figure 2 , the system can be fitted with a supplementary closed position loop, which becomes the main one, while the velocity loop acts as a secondary one.

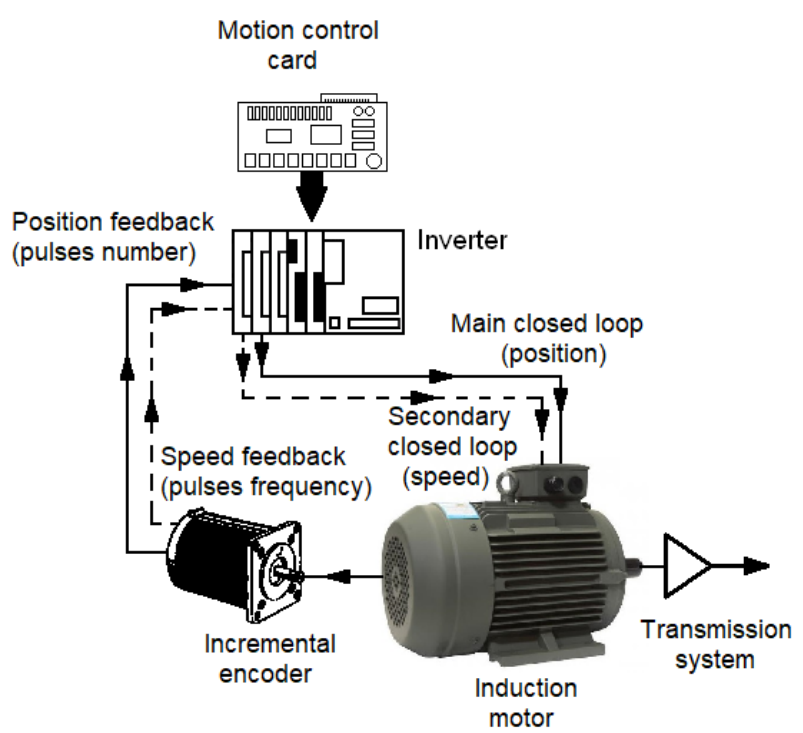

Fig. 2. Closed position and velocity loops with induction motor as actuation device. 


\section{Positioning vs contouring}

Positioning in XY-plane (figure 3a) involves moving the end-effector of the system to reach the points $\mathrm{P}_{1}, \mathrm{P}_{3}$ and $\mathrm{P}_{3}$ with accuracy, without following an imposed toolpath. It is also called point-to-point movement and its main goal is to reach the final programmed points with no (or minimal errors).

In contrast with positioning, contouring (figure $3 \mathrm{~b}$ ) requires the co-ordination of the movements on each axis, in order not only to reach the programmed points, but also to follow an imposed toolpath (trajectory). Usually, following the toolpath is achieved by calculating a significant number of intermediary points by means of an interpolation algorithm.

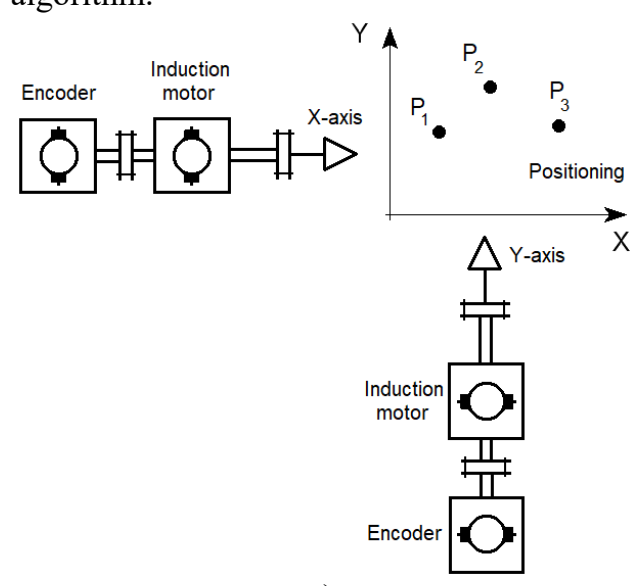

a)

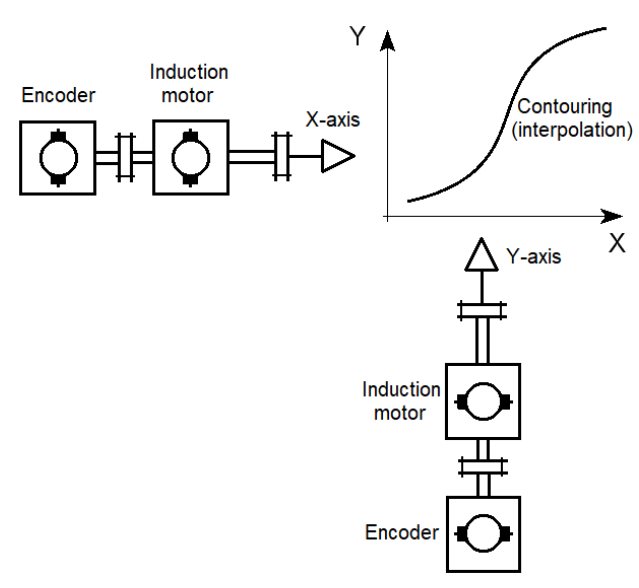

b)

Fig. 3. Positioning a) vs contouring b) in XY-plane.

Contouring usually requires more computing power on the motion control card, making the system more expensive. Low-cost systems are working using the positioning regime. Even the contouring regime allows the system to perform a broader range of tasks, there are still many industrial applications which could be performed by using the positioning regime only.

\section{Generating the inputs}

Control inputs must be provided to the motion control card for the system to reach the programmed positions. The positions within the workspace can be reached using two approaches. In the first approach, the positions are reached through manual control and memorized in a teach-in regime. Subsequently, each position can be called by providing its digital number (on 6 bits) at the digital inputs of the motion control card. The second approach, which was used for the proposed system, uses pulses applied on the digital inputs to control the speed and rotation angles of the motor shaft. Each pulse applied on a digital input issues a command to the motor shaft to rotate with a programmable angular increment of displacement, like in a stepping motor system.

Figure 4 presents the principle of the second approach, where a programmable logic controller (PLC) is used for generating the inputs. Thus, the control inputs are generated on the digital outputs of the PLC and applied at the digital inputs of the motion control card. Using the PLC for that purpose the first has the advantage that complex algorithms required for generating the input pulses (even interpolation algorithms) can be implemented using the programming software of the PLC. 
Inverter + motion control card

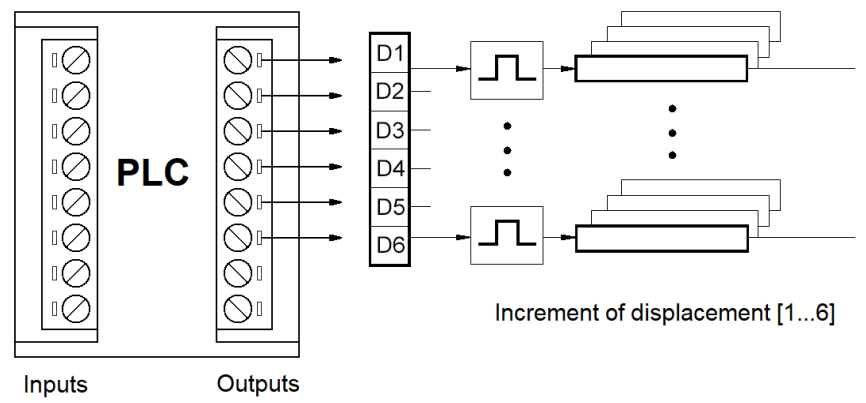

Fig. 4. Generating the input pulses by means of a PLC.

\subsection{Programming the PLC}

A flowchart illustrating the principle of generating the input pulses is presented in figure 5 .

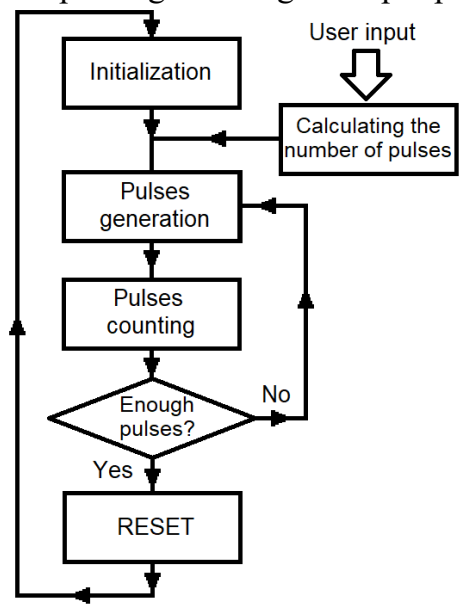

Fig. 5. Flowchart for generating the input pulses.

The "Calculating the number of pulses" section can be tailored by the user according to the needs of the application and/or the working regime (positioning or contouring). In a straightforward approach, the distances on $\mathrm{X}$ and $\mathrm{Y}$ axis to a specific point to be reached should be divided with the transmission system ratio to obtain the necessary number of pulses on each axis.

A simplified programming module made using ladder diagrams, for generating pulses using a digital output of a PLC is presented in figure 6.

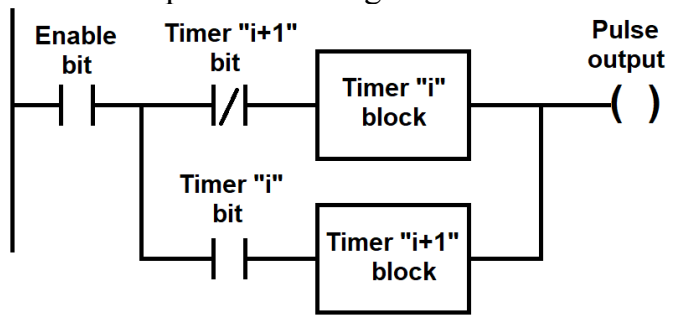

Fig. 6. Programming module for generating input pulses using ladder diagrams. 
The timer blocks presented in figure 6 are simplified, because their structure is hardly dependent of the programming software of the PLC.

\section{Mechanical structure of the system}

The mechanical structure of the system was designed in two variants. The first one, presented in figure 7 a) and b) involves the use of lead screws as transmission systems on both axes.

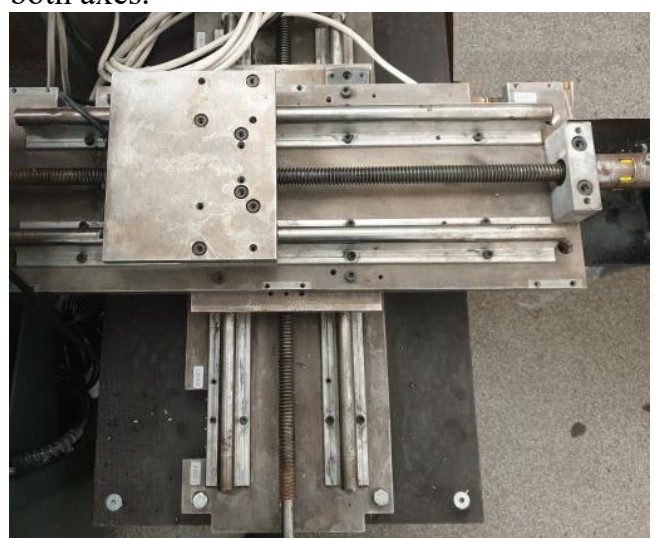

a)

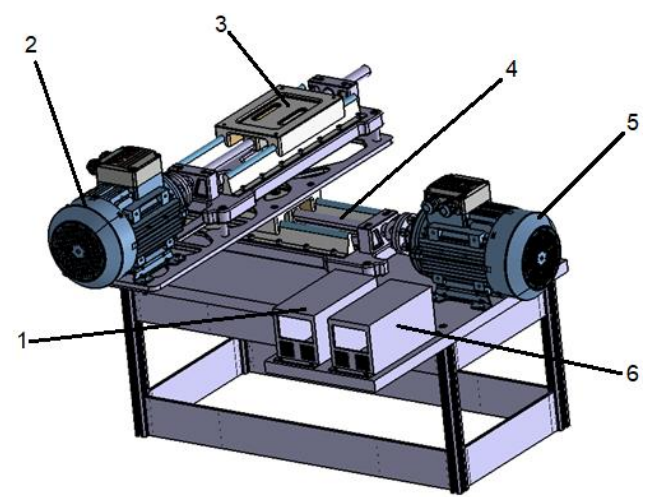

b)

Fig. 7. Mechanical structure of the system with lead screws a) physical system b) 3D model.

The numbers from figure $7 \mathrm{~b}$ ) stand for:

1-inverter for Y-axis, 2-induction motor for Y-axis, 3-slide of the system (end-effector), 4-lead screw of $\mathrm{X}$-axis, 5-induction motor for $\mathrm{X}$-axis, 6-inverter for $\mathrm{X}$-axis.

A second variant of the system presented in figure 8 was also proposed. It was designed according to the H-Bot concept, which uses belts as transmission systems on both axes.

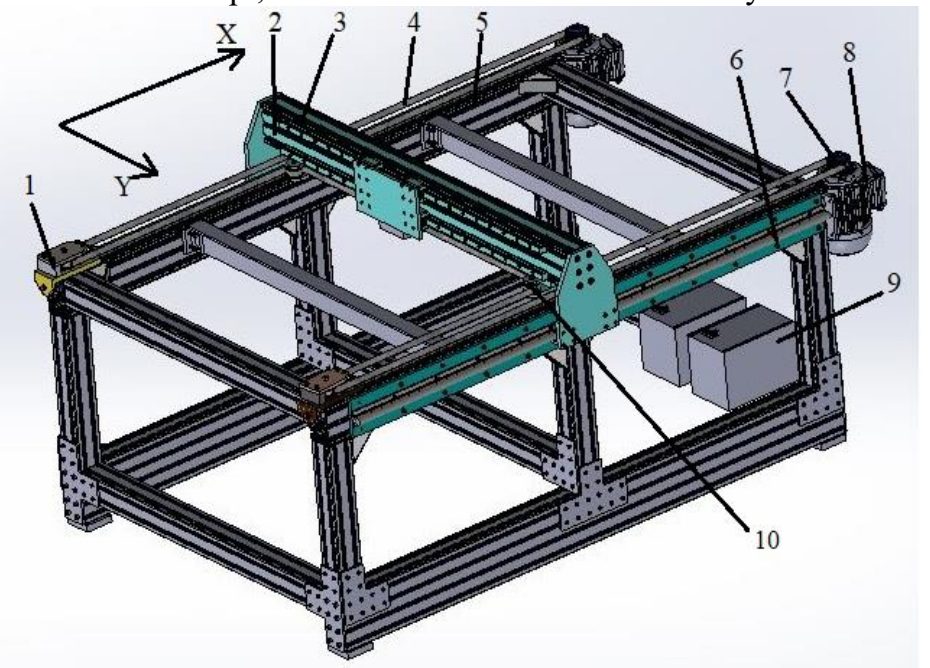

Fig. 8. Mechanical structure of the system with belts - 3D model.

The numbers from figure 8 stand for: 
1-belt tensioning system, 2-Y-axis, 3-linear guideway of $\mathrm{Y}$-axis, 4-belt transmission system, 5-X-axis, 6-linear guideway of $\mathrm{X}$-axis, 7-driving belt drive, 8-induction motor, 9inverter, 10-rollers for changing the belt trajectory.

The second variant of the system is now in the designing stage.

\section{Conclusions}

The work presented in this paper describes a low-cost solution for an industrial positioning system. The main advantages of the system, aside of its low cost are the use of induction motors as actuation devices, which provide high torques and the use of PLC as programming system, which provides the necessary programming flexibility. Future research directions will be oriented to develop the system from a positioning one to a contouring one, by implementing interpolation algorithms at PLC level, while keeping the costs as low as possible.

Acknowledgements: The experimental test unfolded within this research work were partially supported by a grant of the Romanian Ministry of Research and Innovation CCCDI-UEFISCDI, project number PN-III - P1-1.2 - PCCDI-2017-0446/nr. 82PCCDI/2018, within PNCDI III, project title: "Smart manufacturing technologies for advanced production of parts".

\section{References}

1. P. Melin, O. Castillo, Inform. Sciences 170, $2-4$ (2005)

2. M. J. Shi, H. Zhang, X. Liu, Measurement 44, 1 (2011)

3. P. Vas, Sensorless Vector and Direct Torque Control (New York: Oxford Univ. Press, 1998)

4. K. Ohyama, G.M. Asher, M. Sumner, IEEE T. Ind. Electron. 53, 1 (2005)

5. R.-J. Wai, C-C. Chu, IEEE T. Ind. Electron. 54, 1 (2007)

6. Boglietti, A., Cavagnino, M., Lazzari, L. and Pastorelli, M., IEEE T. Ind. Appl. 39, 4 (2003)

7. R. H. Kumar, A. Iqbal, N. C. Lenin, IET Power Electron. 11, 1 (2018)

8. M. Tera, 9th International Technology, Education and Development Conference INTED2015 Proceedings, 5006-5013 (2015)

9. M. Tera, 10th annual International Conference of Education, Research and Innovation ICERI2017 Proceedings, 8167-8172 (2017) 